\title{
BMJ Open Overweight and obesity management strategies in survivors of paediatric acute lymphoblastic leukaemia: a systematic review protocol
}

\author{
Salma Ladhani, ${ }^{1,2}$ Brianna Empringham, ${ }^{1,2}$ Kuan-Wen Wang, ${ }^{1,2,3}$ Carol Portwine, ${ }^{1,4}$ \\ Laura Banfield, ${ }^{5}$ Russell J de Souza, ${ }^{3,6}$ Lehana Thabane, ${ }^{3,6,7,8,9}$ \\ M Constantine Samaan ${ }^{1,2,3,6}$
}

To cite: Ladhani $S$,

Empringham B, Wang K-W, et al. Overweight and obesity management strategies in survivors of paediatric acute lymphoblastic leukaemia: a systematic review protocol. BMJ Open 2018;8:e022530. doi:10.1136/ bmjopen-2018-022530

- Prepublication history and additional material for this paper are available online. To view these files, please visit the journal online (http://dx.doi. org/10.1136/bmjopen-2017022530).

$\mathrm{SL}$ and $\mathrm{BE}$ contributed equally.

Received 22 February 2018

Revised 29 March 2018

Accepted 19 April 2018
Check for updates

For numbered affiliations see end of article.

Correspondence to Dr M Constantine Samaan; samaanc@mcmaster.ca

\section{ABSTRACT}

Introduction Acute lymphoblastic leukaemia is the most common paediatric cancer. Survivors of childhood acute lymphoblastic leukaemia (SALL) are at risk of obesity and related cardiometabolic diseases including type 2 diabetes, hypertension, stroke and cardiovascular events. Therefore, it is important to address obesity in this population as this may help mitigate future cardiometabolic comorbidities. In this systematic review, we aim to assess current treatment strategies including lifestyle interventions, pharmacotherapy and bariatric surgery to manage overweight and obesity in SALL.

Methods and analysis We will search the following databases for primary studies: CINAHL, SPORTDiscus, EMBASE, MEDLINE, PsycINFO, Cochrane Central Register of Controlled Trials, and Cochrane Database of Systematic Reviews. In addition, unpublished primary studies will be searched in ClinicalTrials.gov as well as conference proceedings, presentations, abstracts, editorials and ProQuest Dissertations and Theses A\&l. Reviewers will perform title, abstract, and full-text screening as well as data abstraction and risk of bias assessment independently with a third reviewer to be consulted to resolve disagreements. Searches will be run and updated through May 1st, 2018. The overall quality of the evidence will be determined using the Grading of Recommendations, Assessment, Development, and Evaluation criteria for each outcome. A meta-analysis will be performed if two studies deploying similar interventions, populations, and design and outcomes are identified.

Ethics and dissemination As individual patient data will not be included, we do not require ethics approval. This review will be published in a peer-reviewed journal. PROSPERO registration number CRD42016051031.

\section{BACKGROUND}

Childhood leukaemia is the most common paediatric cancer, ${ }^{1}$ with the most common subtype being acute lymphoblastic leukaemia (ALL). An estimated 2670 children were diagnosed with ALL in the USA in 2014, representing $26 \%$ of all childhood cancer diagnoses. There are close to 380000 survivors of childhood
Strengths and limitations of this study

- This study includes a comprehensive search strategy across several databases to ensure the inclusion of representative studies.

- There is no limitation on language or the time of publication of papers included.

- We plan to use the Grading of Recommendations, Assessment, Development and Evaluation tool to analyse the overall quality of evidence.

- We expect high heterogeneity across studies, which may lead to challenges in performing a meta-analysis.

cancer living in the USA, the majority of whom are over 20 years of age and are leukaemia survivors. ${ }^{1}$

Remarkable advances in the characterisation and treatment of ALL have improved survival rates, with up to $90 \%$ of children diagnosed with ALL surviving beyond 5 years today. ${ }^{23}$ However, survivors of childhood ALL (SALL) are at risk of diseases that influence their long-term outcomes.

One of the common morbidities seen in this population is obesity. ${ }^{45}$ It has been reported that $38 \%$ of SALL are overweight or obese, compared to $31 \%$ of the general paediatric population. ${ }^{4}$ When central obesity is associated with dysglycaemia, hypertension and dyslipidaemia it is referred to as the metabolic syndrome, which is a risk factor for cardiovascular disease and type 2 diabetes. ${ }^{4} 6$ The prevalence of the metabolic syndrome in SALL is slightly higher than that in the general population. ${ }^{7}$

Despite having only modestly higher obesity rates compared to non-cancer controls, SALL have a disproportionately increased risk of insulin resistance, type 2 diabetes, 
cardiovascular disease, stroke and hypertension at a relatively young age. ${ }^{89}$

Several risk factors drive the risk of overweight/obesity and adverse cardiometabolic outcomes in survivors, including treatment with cranial irradiation and corticosteroids, ${ }^{40-13}$ young age at diagnosis $(0-4$ years $),{ }^{11} 14-16$ being overweight or obese at diagnosis ${ }^{15} 16$ and female sex. ${ }^{11} 16-18$

Addressing obesity in SALL is imperative, for the development of obesity in children can lead to obesity in adulthood and this raises the risk of adverse cardiometabolic outcomes. ${ }^{19-21}$

Understanding the risk factors of overweight and obesity in SALL may enable the development of targeted interventions to improve long-term health outcomes by lowering the risk of obesity and its comorbidities. This systematic review aims to assess current interventions to manage overweight and obesity in SALL, and to evaluate their impact on adiposity and associated metabolic comorbidities.

\section{Research question}

In SALL, are lifestyle interventions, pharmacotherapy or bariatric surgery effective in treating overweight and obesity?

\section{OBJECTIVES}

1. To determine the effectiveness of existing interventions to manage overweight and obesity in SALL.

2. If feasible, to perform a meta-analysis of included studies and calculate a precise estimate of the effectiveness of management strategies in SALL.

3. Provide future research directions by identifying gaps in current evidence.

\section{METHODS}

The methodology for this protocol has been established and reported based on the Preferred Reporting Items for Systematic review and Meta-Analysis Protocols (PRISMA-P) guideline (see online supplementary file 1). ${ }^{22} 23$ When changes to the protocol are necessary, we will document the details for the rationale and the change in the reported systematic review.

\section{Patient and public involvement}

Patients and the public were not involved in the design of this review protocol.

\section{Eligibility criteria}

Eligible studies will include SALL diagnosed before the age of 18 years. All subtypes of ALL will be eligible including $\mathrm{T}$ and $\mathrm{B}$ cell leukaemias. Studies involving all interventions targeting overweight and obesity in this population will be included. If there are studies that include other types of childhood cancers, we will extract the data for the SALL population exclusively. If this information is not reported, we will contact the study Principal Investigators to obtain the data.

We will include interventions encompassing lifestyle, pharmacotherapy and bariatric surgery. Lifestyle interventions are defined as treatments that mainly involve exercise and dietary modifications. Pharmacotherapy involves the use of medications for the purpose of weight management. Bariatric surgery is defined as any surgical intervention with the primary goal of treating obesity. All current bariatric surgery procedures will be incorporated into this review including gastric bypass, biliopancreatic diversion with duodenal switch, sleeve gastrectomy and gastric banding. ${ }^{24}$

The eligible study designs include randomised controlled trials (RCTs), quasi RCTs, case-control studies, controlled or uncontrolled studies with before-and-after comparisons, and cross-sectional studies. Review articles, including systematic reviews, will be searched for relevant references if applicable to the research question. Conference proceedings, presentations, abstracts and editorials will be searched for relevant references as well. We will not restrict the language of publication to English. Studies published up to May 1st, 2018 will be included.

\section{OUTCOME MEASURES \\ Primary outcome}

The primary outcome of this review involves comparing changes in body mass index z-score before and after the intervention.

\section{Secondary outcomes}

The secondary outcomes will include changes in adiposity measures including waist circumference, hip circumference, waist-to-hip ratio, waist-to-height ratio and body fat percentage when reported. In addition, we will evaluate changes in insulin sensitivity by measuring the Homeostasis Model Assessment-Insulin resistance (HOMA-IR) or clamp studies, blood pressure $(\mathrm{mmHg})$, non-alcoholic fatty liver disease status by liver ultrasound or elevated transaminases, dyslipidaemia (including cholesterol, triglycerides, high-density lipoprotein and low-density lipoprotein) and obstructive sleep apnoea based on sleep studies.

We will also document any reported adverse effects of the interventions. For lifestyle interventions, these include injuries and back pain. ${ }^{25}{ }^{26}$ For pharmacotherapy, these encompass headaches and insomnia among others. ${ }^{27}$ For bariatric surgery, these include perioperative outcomes and surgical complications. ${ }^{28}$ Other adverse effects will be described if reported.

\section{Search strategy}

When designing the search strategy, we will consult with a Senior Health Sciences Librarian with expertise in designing search strategies for systematic reviews. A sample MEDLINE search strategy is reported in online supplementary file 2. Searches will be conducted in CINAHL, 


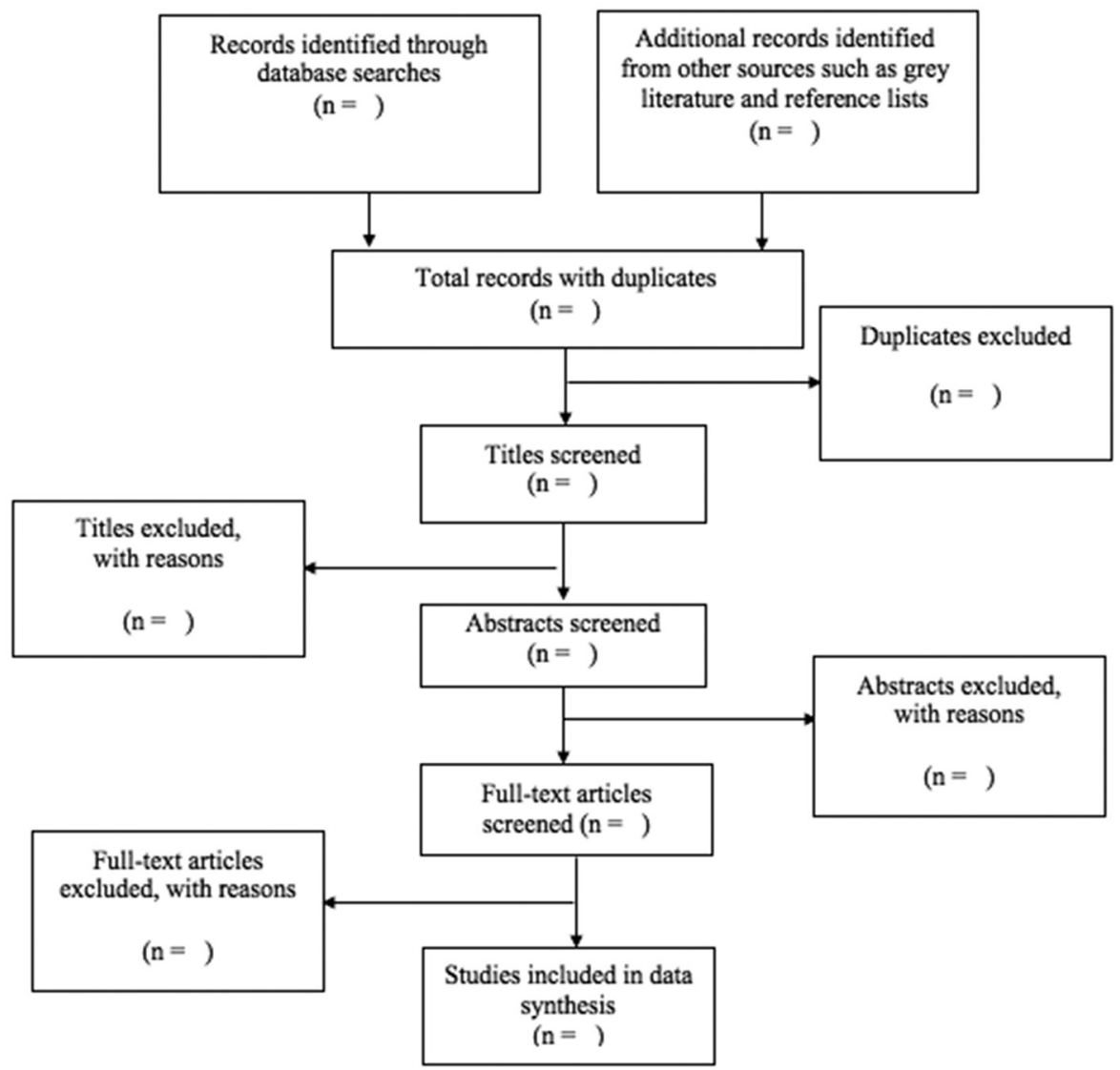

Figure 1 Flow diagram of article screening process.

SPORTDiscus, EMBASE, MEDLINE, PsycINFO, Cochrane Central Register of Controlled Trials, and Cochrane Database of Systematic Reviews. To identify grey literature, we will search ClinicalTrials.gov and ProQuest Dissertations and Theses A\&I. Conference proceedings, presentations, abstracts, and editorials as well as references in reviews including systematic reviews will be examined for eligible articles.

\section{Data management}

We will use EndNote $X 7^{29}$ to remove duplicates from the search results, and references will be exported to an excel file.

\section{Data screening}

We will perform initial screening by two teams, each composed of two reviewers independently for titles and abstracts eligibility. Differences in the included titles and abstracts will be discussed between the reviewers in each team. A third reviewer will address unresolved conflicts at each step of the review process. The reviewers will screen all abstracts, and if there is uncertainty of the eligibility of the abstracts, they will be included. For abstracts deemed eligible for inclusion, the full-text papers will be retrieved for full review. A flow diagram is included here and a populated one will be included in the full review to track the process (figure 1).

\section{Data abstraction}

Pertinent data will be abstracted using a data abstraction form specifically designed for this project. This form will incorporate study details including authors, title, study date, country, study design, inclusion/exclusion criteria, journal of publication and funding details. It will also incorporate participant details including age, sex, ethnicity, age at ALL diagnosis and time from diagnosis to intervention if available.

Other data extracted include treatment types with radiotherapy field (cranial, craniospinal, total body irradiation), radiation type (fractionated vs non-fractionated) and radiation dose (Gy). We will also include chemotherapy protocols, name of medications, dose and duration of treatment. In addition, we will collect data on bone marrow transplantation, and medical and metabolic comorbidities will be abstracted.

The targeted obesity intervention details will be abstracted including study design, intervention details, duration and follow-up periods and any reported side effects.

Details of the statistics performed and results will be abstracted. In order to retrieve incomplete data, we will attempt to contact the Principal Investigators for each project. Outcomes will be summarised along with the overall conclusions and any confounding factors. 


\section{Risk of bias and quality assessment}

The risk of bias assessment tool created by the Cochrane Collaboration will be used to evaluate the risk of bias for RCTs. ${ }^{30}$ The studies will be rated as having a high, low or unclear risk of bias based on the validity of sequence generation, allocation concealment, blinding, data completeness, selective reporting and other sources of bias.

Non-randomised trials will be assessed using the Risk of Bias in Non-Randomised Studies-of Interventions (ROBINS-I) tool. ${ }^{31}$ This tool considers confounding variables and selection bias at the preintervention stage. The following confounding factors will be considered when using this tool: age at time of enrolment in the intervention, age at diagnosis, pubertal stage, sex, baseline body composition, treatments received for ALL, years since end of therapy and comorbidities such as metabolic syndrome and hormonal deficiencies. At the intervention stage, misclassification of intervention group will be evaluated. Lastly, deviation from intended interventions, missing data, standardised methods of outcome measures and selective reporting will be assessed at the postintervention stage.

In uncontrolled before and after studies, the risk of bias will be assessed using the University of Alberta Evidencebased Practice Center checklist. ${ }^{32}$ This checklist evaluates patient enrolment, incomplete data and standardised approach to outcomes to determine the risk of bias.

The overall quality of the body of evidence will be evaluated with the Grading of Recommendations, Assessment, Development and Evaluation (GRADE) approach. ${ }^{33}$ We will consider the quality of the evidence and the magnitude of the effect to determine the overall strength of the meta-analysis findings if it is feasible to do so. The determinants of quality include risk of bias, inconsistency, indirectness, imprecision and publication bias. The overall strength of evidence will be classified as high, moderate, low or very low for each outcome measure.

\section{Data analysis}

We will include the summary and details of each relevant study in our analysis. If two or more studies of similar interventions, populations, study design, and outcomes are identified, a meta-analysis will be conducted. Categorical outcomes will be reported as odds ratios, and continuous variables will be reported as mean differences with $95 \%$ CI.

We will perform the meta-analysis with a random effects model if more than ten studies can be included using Review Manager software. ${ }^{34}$ We are expecting high heterogeneity across studies, and this random effects model is the preferred method in this case.$^{35}$ Otherwise, we will present results from both random and fixed effects models. ${ }^{36}$

Heterogeneity across studies will be evaluated with inconsistency index $\left(\mathrm{I}^{2}\right)$ and $\mathrm{p}$ values from $\mathrm{X}^{2}$ test for homogeneity, with $\mathrm{I}^{2}>75 \%$ and a $\mathrm{p}$ value of $<0.10$ representing considerable heterogeneity. ${ }^{37}$

If appropriate, we will perform subgroup meta-analysis by sex, as female SALL have been reported to have higher risk of obesity than male SALL and may respond to overweight and obesity management strategies differently. ${ }^{116-18}$
If possible, subgroup analyses will also be done based on treatment modalities, including those treated for a relapse or receiving a bone marrow transplantation. We will determine publication bias by creating a funnel plot if ten or more studies are included. ${ }^{38}$ We will use Egger's test, performed with PASW V.18 statistical package, ${ }^{39}$ and visual inspection to determine the plot asymmetry.

Alternatively, we will estimate publication bias by considering the number of relevant conference abstracts without published articles. If a meta-analysis is not appropriate, a summary table with narrative description will be reported.

\section{DISCUSSION}

Improvements in treatments and supportive care have led to an increased survival in children diagnosed with ALL. However, comorbidities in SALL are high and affect the quality of life and long-term outcomes. The increased rates of obesity in this population represent a potential area of need for intervention to help improve cardiometabolic outcomes in SALL.

Around $50 \%$ of SALL have at least one chronic health condition compared to $37.8 \%$ of sibling controls. ${ }^{40}$ It is vitally important to understand if effective overweight and obesity interventions are available for SALL, as ALL treatment is associated with high cure rates. As survivors are forced to live longer with the burden of their cancer history, this study will summarise the current scientific understanding of interventions that target overweight and obesity in SALL, and will identify gaps in current research to guide future study design to improve outcomes.

\section{ETHICS AND DISSEMINATION}

We will not include individual patient data in this review. Therefore, ethics approval is not required, and there are no further ethical and safety considerations. The dissemination plan for this review is to be published in a peer-reviewed journal.

\section{Author affiliations}

${ }^{1}$ Department of Pediatrics, McMaster University, Hamilton, Ontario, Canada ${ }^{2}$ Division of Pediatric Endocrinology, McMaster Children's Hospital, Hamilton, Ontario, Canada

${ }^{3}$ Medical Sciences Graduate Program, McMaster University, Hamilton, Ontario, Canada

${ }^{4}$ Division of Pediatric Hematology/Oncology, McMaster Children's Hospital, Hamilton, Ontario, Canada

${ }^{5}$ Health Sciences Library, McMaster University, Hamilton, Ontario, Canada ${ }^{6}$ Department of Health Research Methods, Evidence and Impact, McMaster University, Hamilton, Ontario, Canada

${ }^{7}$ Department of Anesthesia, McMaster University, Hamilton, Ontario, Canada ${ }^{8}$ Centre for Evaluation of Medicines, St. Joseph's Health Care, Hamilton, Ontario, Canada

${ }^{9}$ Biostatistics Unit, St Joseph's Healthcare, Hamilton, Ontario, Canada

Acknowledgements We would like to thank Ms. E. Danielle Sims for assistance in addressing the editorial comments.

Contributors MCS is the guarantor. Research question was defined by SL, BE, CP, RJdS, LT and MCS. SL, BE, KWW, CP, LB, RJdS, LT and MCS contributed to the development of search strategy and determination of the eligibility criteria. SL, 
BE, LB, KWW and MCS designed the data abstraction form. RJdS and LT provided methodological support for this review. SL, BE, KWW, CP and MCS wrote the first draft of the manuscript, and the final version was reviewed and approved by all authors.

Funding MCS was funded by the Pediatric Oncology Group of Ontario (POGO) Research Unit, and the Hamilton Health Sciences and Foundation. KWW was funded by the Ontario Graduate Scholarship Program, and the Canadian Institutes of Health Research (CIHR) Canada Graduate Scholarship-Masters. Design of the systematic review is independent of the funding agencies.

Competing interests None declared.

Patient consent Not required.

Provenance and peer review Not commissioned; peer reviewed for ethical and funding approval prior to submission.

Open access This is an open access article distributed in accordance with the terms of the Creative Commons Attribution (CC BY 4.0) license, which permits others to distribute, remix, adapt and build upon this work, for commercial use, provided the original work is properly cited. See: http://creativecommons.org/ licenses/by/4.0/

(c) Article author(s) (or their employer(s) unless otherwise stated in the text of the article) 2018. All rights reserved. No commercial use is permitted unless otherwise expressly granted.

\section{REFERENCES}

1. Ward E, DeSantis C, Robbins A, et al. Childhood and adolescent cancer statistics, 2014. CA Cancer J Clin 2014;64:83-103.

2. Pui $\mathrm{CH}$, Evans WE. Treatment of acute lymphoblastic leukemia. $N$ Engl J Med 2006;354:166-78

3. Pulte D, Gondos A, Brenner H. Trends in 5- and 10-year survival after diagnosis with childhood hematologic Malignancies in the United States, 1990-2004. JNCI 2008;100:1301-9.

4. Chow EJ, Pihoker $\mathrm{C}$, Hunt $\mathrm{K}$, et al. Obesity and hypertension among children after treatment for acute lymphoblastic leukemia. Cancer 2007;110:2313-20.

5. Withycombe JS, Smith LM, Meza JL, et al. Weight change during childhood acute lymphoblastic leukemia induction therapy predicts obesity: a report from the Children's Oncology Group. Pediatr Blood Cancer 2015:62:434-9.

6. Expert Panel on Detection, Evaluation, and Treatment of High Blood Cholesterol in Adults. Executive summary of the third report of The National Cholesterol Education Program (NCEP) Expert Panel on Detection, Evaluation, And Treatment of High Blood Cholesterol In Adults (Adult Treatment Panel III). JAMA 2001;285:2486-97.

7. Kourti M, Tragiannidis A, Makedou A, et al. Metabolic syndrome in children and adolescents with acute lymphoblastic leukemia after the completion of chemotherapy. J Pediatr Hematol Oncol 2005;27:499-501.

8. Oeffinger KC. Are survivors of acute lymphoblastic leukemia (ALL) at increased risk of cardiovascular disease? Pediatr Blood Cancer 2008;50(2 Suppl):462-7.

9. Oeffinger KC, Adams-Huet B, Victor RG, et al. Insulin resistance and risk factors for cardiovascular disease in young adult survivors of childhood acute lymphoblastic leukemia. J Clin Oncol 2009;27:3698-704.

10. Garmey EG, Liu Q, Sklar CA, et al. Longitudinal changes in obesity and body mass index among adult survivors of childhood acute lymphoblastic leukemia: a report from the childhood cancer survivor study. J Clin Oncol 2008;26:4639-45.

11. Oeffinger KC, Mertens AC, Sklar CA, et al. Obesity in adult survivors of childhood acute lymphoblastic leukemia: a report from the Childhood Cancer Survivor Study. J Clin Oncol 2003;21:1359-65.

12. Sklar CA, Mertens AC, Walter A, et al. Changes in body mass index and prevalence of overweight in survivors of childhood acute lymphoblastic leukemia: role of cranial irradiation. Med Pediatr Oncol 2000;35:91-5.

13. van Beek RD, de Muinck Keizer-Schrama SM, Hakvoort-Cammel $\mathrm{FG}$, et al. No difference between prednisolone and dexamethasone treatment in bone mineral density and growth in long term survivors of childhood acute lymphoblastic leukemia. Pediatr Blood Cancer 2006;46:88-93.

14. Dalton VK, Rue M, Silverman LB, et al. Height and weight in children treated for acute lymphoblastic leukemia: relationship to CNS treatment. J Clin Oncol 2003;21:2953-60.
15. Razzouk BI, Rose SR, Hongeng S, et al. Obesity in survivors of childhood acute lymphoblastic leukemia and lymphoma. J Clin Oncol 2007;25:1183-9.

16. Withycombe JS, Post-White JE, Meza JL, et al. Weight patterns in children with higher risk ALL: A report from the Children's Oncology Group (COG) for CCG 1961. Pediatr Blood Cancer 2009;53:1249-54.

17. Kohler JA, Moon RJ, Wright S, et al. Increased adiposity and altered adipocyte function in female survivors of childhood acute lymphoblastic leukaemia treated without cranial radiation. Horm Res Paediatr 2011;75:433-40.

18. Odame I, Reilly JJ, Gibson BE, et al. Patterns of obesity in boys and girls after treatment for acute lymphoblastic leukaemia. Arch Dis Child 1994;71:147-9.

19. Bray GA. Predicting obesity in adults from childhood and adolescent weight. Am J Clin Nutr 2002;76:497-8.

20. Guo SS, Wu W, Chumlea WC, et al. Predicting overweight and obesity in adulthood from body mass index values in childhood and adolescence. Am J Clin Nutr 2002;76:653-8.

21. Simmonds M, Llewellyn A, Owen CG, et al. Predicting adult obesity from childhood obesity: a systematic review and meta-analysis. Obes Rev 2016;17:95-107.

22. Moher D, Shamseer L, Clarke M, et al. Preferred reporting items for systematic review and meta-analysis protocols (PRISMA-P) 2015 statement. Syst Rev 2015;4:1.

23. Moher D, Stewart L, Shekelle P. Implementing PRISMA-P: recommendations for prospective authors. Syst Rev 2016;5:15.

24. Buchwald H, Oien DM. Metabolic/bariatric surgery Worldwide 2008. Obes Surg 2009;19-1605-11.

25. Ried-Larsen M, Christensen R, Hansen KB, et al. Head-to-head comparison of intensive lifestyle intervention (U-TURN) versus conventional multifactorial care in patients with type 2 diabetes: protocol and rationale for an assessor-blinded, parallel group and randomised trial. BMJ Open 2015;5:e009764.

26. Yang Z, Scott CA, Mao C, et al. Resistance exercise versus aerobic exercise for type 2 diabetes: a systematic review and meta-analysis. Sports Med 2014;44:487-99.

27. Kang JG, Park CY. Anti-Obesity drugs: a review about their effects and safety. Diabetes Metab J 2012;36:13-25.

28. Hopkins JC, Howes $\mathrm{N}$, Chalmers $\mathrm{K}$, et al. Outcome reporting in bariatric surgery: an in-depth analysis to inform the development of core outcome set, the BARIACT Study. Obes Rev 2015;16:88-106.

29. EndNote [Computer program]. Version 7.7.1 [program]: Clarivate Analytics. 2016.

30. Higgins JPT, Altman DG, Sterne JAC. Chapter 8: Assessing risk of bias in included studies. In: Higgins JPT, Green S, Cochrane handbook for systematic review of interventions Version 510. eds: The Cochrane Collaboration, 2011. (accessed Mar 2011).

31. Sterne JA, Hernán MA, Reeves BC, et al. ROBINS-I: a tool for assessing risk of bias in non-randomised studies of interventions. BMJ 2016;355:i4919.

32. Seida JC, Schouten JR, Mousavi SS, et al. Methods. Comparative effectiveness of nonoperative and operative treatment for rotator cuff tears. Rockville (MD): Agency for Healthcare Research and Quality (US), 2010:2

33. Atkins D, Best D, Briss PA, et al. Grading quality of evidence and strength of recommendations. BMJ 2004;328:1490.

34. Review Manager. Computer program]. Version 5.3. [program). Copenhagen: The Nordic Cochrane Centre, The Cochrane Collaboration, 2014

35. Villar J, Mackey ME, Carroli G, et al. Meta-analyses in systematic reviews of randomized controlled trials in perinatal medicine: comparison of fixed and random effects models. Stat Med 2001;20:3635-47.

36. Borenstein M, Hedges LV, Higgins JP, et al. A basic introduction to fixed-effect and random-effects models for meta-analysis. Res Synth Methods 2010;1:97-111.

37. Deeks JJ, Higgins JPT, Altman DG. Chapter 9: Analysing data and undertaking meta-analyses. In: Higgins JPT, Green S, eds. Cochrane handbook for systematic reviews of interventions version 510: The Cochrane Collaboration, 2011. (accessed Mar 2011).

38. Sterne JAC, Egger M, Moher D. Chapter 10: Addressing reporting biases. In: Higgins JPT, Green S, eds. Cochrane handbook for systematic reviews of intervention version 510: The Cochrane Collaboration, 2011. (accessed Mar 2011).

39. Alexander RW. President's address. Common mechanisms of multiple diseases: why vegetables and exercise are good for you. Trans Am Clin Climatol Assoc 2010;121:1-20.

40. Mody R, Li S, Dover DC, et al. Twenty-five-year follow-up among survivors of childhood acute lymphoblastic leukemia: a report from the Childhood Cancer Survivor Study. Blood 2008;111:5515-23. 